\title{
Aspectos para integrar el enfoque de resultados de aprendizaje en el diseño curricular universitario
}

\author{
Aspects to be considered to integrate the learning outcomes approach \\ into university curricular design
}

Aspectos a considerar para integrar o enfoque de resultados de aprendizagem no desenho curricular universitário

\author{
Livieth Gamboa Solano \\ Universidad de Costa Rica \\ Cartago, Costa Rica \\ livieth.gamboa@ucr.ac.cr \\ (D) https://orcid.org/0000-0002-1498-0172 \\ María Gabriela Guevara Mora \\ Universidad de Costa Rica \\ Cartago, Costa Rica \\ gabriela.guevara@ucr.ac.cr \\ (D) https://orcid.org/0000-0002-0287-0793
Álvaro Mena
Cartago, Costa Rica
alvaro.mena@ucr.ac.cr \\ Universidad de Costa Rica \\ (D) https://orcid.org/0000-0003-0612-0532 \\ Ana Cristina Umaña Mata \\ Universidad Estatal a Distancia \\ San José, Costa Rica \\ acumanam@uned.ac.cr \\ (D) https://orcid.org/0000-0002-7998-1061
}

Recibido - Received - Recebido: 19/03 / 2021 Corregido - Revised - Revisado: 06 / 06 / 2021 Aceptado - Accepted - Aprovado: 10 / 06 / 2021

DOl: https://doi.org/10.22458/ie.v23i34.3474

URL: https://revistas.uned.ac.cr/index.php/innovaciones/article/view/3474

\begin{abstract}
Resumen: El presente artículo tiene como finalidad presentar los hallazgos de la investigación realizada para identificar aspectos importantes, con el fin de considerarlos al utilizar el enfoque de resultados de aprendizaje en el currículo universitario. El diseño de la investigación fue de corte cualitativo y, se considera de tipo exploratorio; pues resultó el primer acercamiento al tema. Se trabajó con el método de revisión de literatura y entrevista a personas expertas en el campo. A lo largo del artículo se abordan temáticas asociadas a este enfoque como: redacción, coherencia de los resultados de aprendizaje en relación con los componentes curriculares, uso de taxonomías para la clasificación de los niveles de conocimiento, mapeo curricular, beneficios y retos.

Como principales resultados se halla que tanto a nivel nacional como internacional se conoce del enfoque de resultados de aprendizaje y existen propuestas educativas que lo incluyen, específicamente, en el perfil, la metodología y la evaluación. Sin embargo, a nivel de concreción curricular en asignaturas o cursos, los esfuerzos son aislados y falta mayor tratamiento del tema. Por otra parte, se evidencia que es fundamental contar con una taxonomía para la redacción de los resultados de aprendizaje, las de Bloom, Bloom revisado y SOLO son las más utilizadas.
\end{abstract}

Palabras clave: Aprendizaje, Educación superior, Evaluación, Plan de estudios, Taxonomía, Educación 


\begin{abstract}
The purpose of this article is to present the findings of the research carried out to identify important aspects that should be considered when using the learning outcomes approach in the university curriculum. The research design was qualitative and is considered exploratory, since it was the first approach to the subject. We worked with the method of reviewing the literature and interviewing experts in the field. Throughout the article, topics associated with this approach are addressed, such as writing, coherence of learning results with regard to curricular components, use of taxonomies for classifying levels of knowledge, curriculum mapping, benefits and challenges. As main results, it is found that both nationally and internationally the approach is known and that there are educational proposals that specifically include it in the profile, methodology and evaluation. However, at the level of curricular implementation in subjects or courses, the efforts are isolated and there is a lack of further treatment of the subject. On the other hand, it is evident that it is essential to have a taxonomy for the drafting of the learning results, being those of Bloom, Bloom revised and SOLO the most used.
\end{abstract}

Keywords: Learning, Higher Education, Assessment, Curriculum, Taxonomy

Resumo: $O$ objetivo deste artigo é apresentar os resultados da pesquisa realizada para identificar aspectos importantes que devem ser considerados ao utilizar a abordagem dos resultados do aprendizado no currículo universitário. O desenho da pesquisa foi qualitativo e é considerado exploratório, já que foi a primeira abordagem ao assunto. Trabalhamos com o método de revisão de literatura e entrevistas com especialistas na área. Ao longo do artigo, são abordados tópicos associados a este enfoque, tais como redação, coerência dos resultados de aprendizagem em relação aos componentes curriculares, uso de taxonomias para a classificação dos níveis de conhecimento, mapeamento curricular, benefícios e desafios. Como principais resultados, constata-se que tanto nacional como internacionalmente a abordagem é conhecida e que existem propostas educacionais que a incluem, especificamente no perfil, metodologia e avaliação. Entretanto, ao nível da concretização curricular em disciplinas ou cursos, os esforços são isolados e há uma falta de maior tratamento do tema. Por outro lado, é evidente que é essencial ter uma taxonomia para a redação dos resultados do aprendizado, sendo a Bloom's, a revista Bloom's e a SOLO as mais utilizadas.

Palavras chave: Aprendizagem Educação Superior, Avaliação, Plano de Estudos, Taxonomia

\title{
INTRODUCCIÓN
}

Las transformaciones derivadas de los procesos de globalización y la denominada "sociedad del conocimiento" han sido catalizadores para que se lleven a cabo iniciativas tendientes a promover la movilidad, el reconocimiento y la transparencia de la educación superior entre los países. Un ejemplo de ello es la Declaración de Boloña, en 1999, en la cual varios ministros y ministras de educación de las naciones europeas coincidieron en múltiples líneas de acción que incluían: la adopción de un sistema de educación superior basado en dos ciclos (bachillerato y maestría); el reconocimiento de los grados y diplomas en el área de educación europea con fines laborales o de estudio; acceso a oportunidades laborales de las personas graduadas; movilidad estudiantil y docente; enlace del aprendizaje para la vida con la educación superior; y, promoción de la educación superior en el mercado mundial (Thompson, 2011).

En el 2005, como resultado del acuerdo expuesto en el párrafo anterior, se concretó el marco de cualificaciones del Área de Educación Superior Europea, el cual define un conjunto de resultados de aprendizaje para cada uno de los tres ciclos de la educación superior, de manera que se varíe el modelo tradicional de la enseñanza centrada en la persona docente, por el aprendizaje centrado en el estudiantado. A estos resultados se les conoce como los descriptores de Dublín, los cuales son sentencias genéricas que se adaptan a múltiples disciplinas y marcos nacionales de cualificación, y, expresan expectativas típicas de logros y habilidades asociadas con las cualificaciones que representan el final de cada uno de los ciclos de Bolonia (Bologna Working Group on Qualifications Frameworks, 2005). Estos descriptores fueron redactados en términos de las siguientes categorías: conocimiento y comprensión, aplicación de conocimientos y comprensión, capacidad para emitir juicios, capacidad de comunicar, habilidades de aprendizaje.

A nivel de la región centroamericana, en la cual se circunscribe esta investigación, los esfuerzos se han orientado a buscar opciones que permitan contar con requerimientos mínimos en cuanto a perfiles de las personas graduadas y sus estándares de cualificación. En ese sentido, cobra relevancia el documento Marco de Cualificación para la Educación Superior Centroamericana (MCESCA), el cual agrupa, de 
manera sustancial, los resultados de aprendizaje que el estudiantado debe lograr, en los diversos niveles académicos que se ofrecen en la educación superior: pregrado, grado y posgrado (Consejo Superior Universitario Centroamericano, 2018).

Dado que el MCESCA efectúa un planteamiento de diseñar los planes de estudio por resultados de aprendizaje y, desde el Consejo Nacional de Rectores de Costa Rica (CONARE) existe un interés por implementarlo, nace la propuesta de hacer una primera aproximación de los posibles aspectos de índole curricular que deben considerarse tanto en el diseño como en la implementación de ofertas educativas bajo este enfoque. Sin embargo, a partir de la revisión bibliográfica realizada, se encontró que no hay información suficiente para determinar con claridad los requisitos y las características para la atención de los componentes curriculares, presentes en el diseño de planes de estudios, desde el enfoque de resultados de aprendizaje. De esta manera, el problema surge para atender este faltante; pues la documentación teórica no resulta suficiente para orientar el proceso requerido para la incorporación de este enfoque.

De acuerdo con lo anterior, los objetivos de la presente investigación son: determinar las consideraciones relacionadas con el diseño curricular de planes de estudio y asignaturas o cursos, desde el enfoque de resultados de aprendizaje, y, por medio del criterio de expertos, explorar cómo han incorporado este enfoque en los procesos de diseño curricular universitario.

En cuanto a los referentes teóricos de esta investigación, al revisar la literatura, se evidencia una diversidad de enfoques en virtud de la definición de resultados de aprendizaje, en su mayoría con un énfasis en el estudiantado. CEDEFOP (2017), Bologna Working Group on Qualifications Frameworks (2005), Australian Qualifications Framework (2013) y Consejo Superior Universitario Centroamericano (2018) resaltan que los resultados de aprendizaje son declaraciones de lo que se espera que el estudiantado conozca, comprenda o sea capaz de hacer al terminar un proceso de aprendizaje. Relacionado con lo anterior, CEDEFOP (2017), además, cataloga los resultados de aprendizaje como sentencias o expresiones de intención presentes en los marcos de cualificaciones, en estándares de cualificación y los planes de estudio.

En ese sentido, resulta importante señalar que diversos autores e instituciones utilizan el término competencias en lugar de resultados de aprendizaje; sin embargo, ambos términos se aplican de muy diversas maneras, al punto que la frontera entre ambos no siempre está clara y depende del contexto en que se utilice, lo cual provoca confusión y contradicción en algunas ocasiones.

Bohlinger (2008, p. 106) define las competencias como: la "[...] demostrada capacidad para utilizar conocimientos, destrezas y habilidades" y, coincide con Kennedy y McCarthy (2016), en que la competencia se describe en términos de responsabilidad y autonomía.

Por otro lado, la Comisión Europea (2009) considera que las competencias son parte de los resultados de aprendizaje y define competencias como: "la capacidad demostrada para utilizar conocimientos, destrezas y habilidades personales, sociales y metodológicas, en situaciones de trabajo o estudio y en el desarrollo profesional y personal" (p. 11). Por ende, la competencia puede entenderse como el logro y la validación de los resultados de aprendizaje expresada en la capacidad de la persona para aplicar, de manera autónoma, conocimientos y habilidades en la sociedad, en su proceso educativo y el trabajo.

Así, ante la necesidad de aclarar la confusión entre ambos términos, la Agencia Nacional de Evaluación de la Calidad y Acreditación (s.f.) considera que los resultados del aprendizaje son concreciones de las competencias para un determinado nivel y además, son el resultado global del proceso de enseñanza-aprendizaje.

Otro término que merece atención es el concepto de objetivo, el cual según Kennedy (2006, p. 20) consiste en "un enunciado específico en relación a lo que se va a enseñar, es decir, señala una de las áreas específicas que el profesor pretende cubrir en un bloque de enseñanza." Puede notarse que la intención 
de un objetivo es distinta a la de un resultado de aprendizaje, por cuanto el primero se enfoca en el proceso de enseñanza y el segundo en el proceso de aprendizaje.

Por otra parte, el diseño curricular se comprende como un proceso primordial para el planteamiento del currículo (Clemente Linuesa, 2010); pues conlleva la posible articulación de los diferentes componentes curriculares que deben considerarse en la elaboración de una propuesta formativa. Cuando se trabaja por resultados de aprendizaje, el planteamiento del currículo se hace desde el perfil de salida (se refiere al tipo de profesional que se espera al finalizar los estudios), esto para los documentos relacionados con planes de estudio. Mientras que, en la descripción de las asignaturas o cursos, serían detallados de forma específica, según se espera que el estudiantado pueda desarrollar en su experiencia de aprendizaje.

Toda propuesta educativa independiente del modelo y la posición epistemológica y curricular que asuma para su implementación, debe contemplar un conjunto de elementos básicos, los cuales orientarán el proceso de enseñanza-aprendizaje. Estos elementos han sido denominados de diferentes maneras. Coll (1997), Bolaños y Molina (1997) consideran que el nombre más indicado para su denominación sería la de "elementos curriculares", por su vinculación en los procesos propios de la organización del currículo. De esta manera, coinciden en la necesidad de contemplarlos para el desarrollo exitoso de una propuesta educativa. Según Coll (1997) responden a las siguientes preguntas esenciales:

- ¿Qué se quiere enseñar?, lo cual refiere a los contenidos.

- ¿Para qué se quiere enseñar?, se enfoca en los objetivos.

- ¿Cómo se va a enseñar?, busca dar respuesta a la metodología de enseñanza y las actividades de aprendizaje.

- ¿Cómo se va a evaluar? se enfoca en la delimitación de aquellas actividades evaluativas.

Cuando se trabaja por resultados de aprendizaje, los objetivos de la asignatura o curso deben plantearse mediante aquellos resultados de aprendizaje que se esperan del estudiantado al finalizar sus estudios, lo cual impacta en el diseño de la metodología (actividades) y la evaluación. Posner (2007), refiere la importancia de considerar otros aspectos que permiten esa vinculación entre los elementos, tal es el caso del fundamento o filosofía educativa en que se enmarca el currículo, lo mismo que la secuenciación y orden en que serán desarrollados los objetivos y los contenidos. También se toman en cuenta aspectos vinculados con las características sociodemográficas y educativas del estudiantado, así como la infraestructura y tecnología que se dispone para el desarrollo del proceso educativo. En síntesis, la manera en que sean conceptualizados, el énfasis e importancia que se den a cada uno de esos aspectos y la forma en que se defina su atención, marca la diferencia entre un proyecto educativo y otro.

Para que realmente se puedan visualizar los resultados de aprendizaje en el proceso formativo, se requiere efectuar un trabajo de alineamiento. Cohen (1987) es quizás uno de los autores que primero se refirió al término, como la congruencia que debe darse entre los diferentes componentes del currículo.

En el caso del alineamiento constructivo, esta teoría la planteó John Biggs (citado por Brabrand y Dahl, 2007b) y se refiere al hecho de que, en un buen diseño curricular, los resultados de aprendizaje establecidos, las tareas de aprendizaje/enseñanza y las actividades de evaluación convergen de manera coherente hacia el logro de los resultados del aprendizaje. Según Alfauzan y Tarchouna (2017) el profesorado debe asegurar la coherencia entre los resultados de aprendizaje establecidos en sus cursos, las actividades de enseñanza/aprendizaje que diseñan o seleccionan y las tareas de evaluación que les posibilita medir el rendimiento estudiantil de conformidad con los resultados de aprendizaje establecidos.

Wijngaards-de Meij y Merx (2018) también consideran crucial el alineamiento curricular para el logro de los objetivos de aprendizaje, pero determinaron problemas prácticos a nivel de la educación superior. Entre ellos, se señalan la falta de conciencia del estudiantado sobre su posición dentro del currículo y 
proponen la implementación de herramientas de mapeo curricular digitales, como medio para visibilizar las trayectorias de aprendizaje a través del currículo.

La figura 1 ilustra el alineamiento en un curso, según lo que indica Brabrand y Dahl (2007b), puede observarse que en el escenario a) las intenciones del proceso educativo están desajustadas con respecto a las actividades de evaluación y de aprendizaje que se le proponen al estudiantado. Lo anterior se puede notar en el uso de verbos distintos, lo que provoca que en la evaluación se midan resultados de aprendizaje que se apartan de la intención educativa y ocasiona que el estudiantado se enfoque, principalmente, en los resultados de aprendizaje con los que se realizarán las pruebas. Por otro lado, en el escenario b) tanto las intenciones educativas, representadas en los resultados de aprendizaje previstos, como las actividades de enseñanza/aprendizaje y las evaluativas están alineadas. Esto último es muy positivo porque el estudiantado tiene un panorama, en el cual los resultados de aprendizaje previstos le guiarán en todo su proceso, lo que repercutirá en su compromiso y motivación (Brabrand \& Dahl, 2007b).

Figura 1. Un curso desalineado versus un curso alineado

a) Curso desalineado

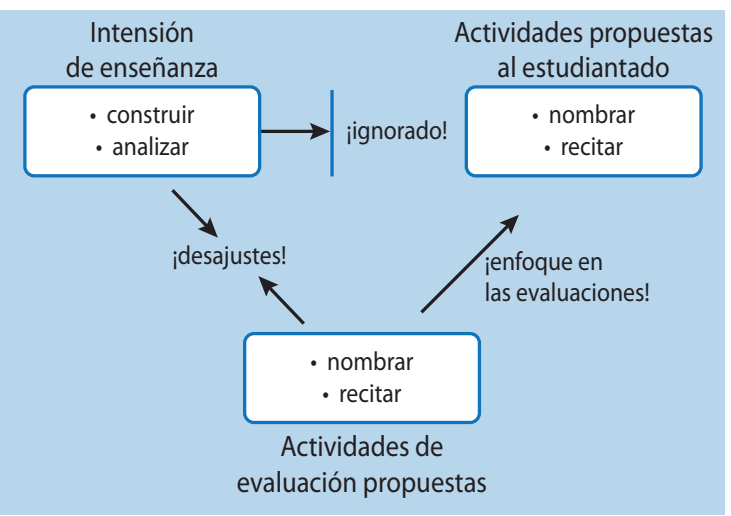

a) Curso alineado

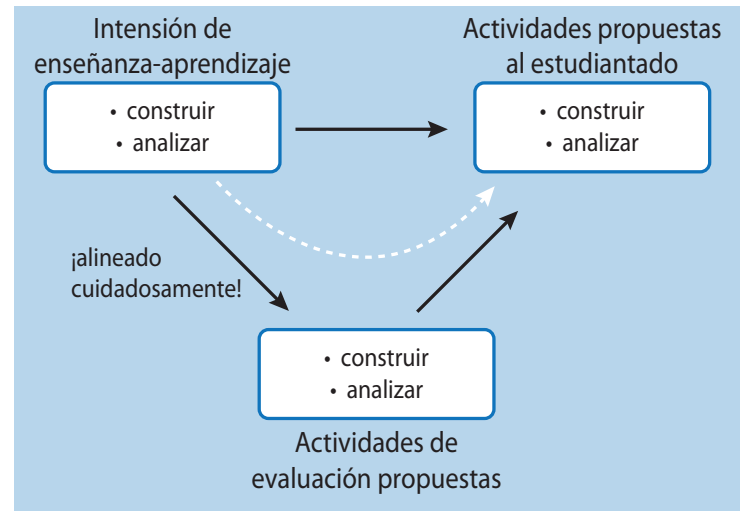

Fuente: Adaptado de Brabrand y Dahl (2007b, p. 5).

Para llevar a cabo el alineamiento de manera adecuada, se puede emplear el mapeo curricular como una herramienta para "[...] discernir si los diferentes componentes curriculares se alinean y, si no, qué ajustes se pueden hacer" Kopera-Frye, Mahaffy y Svare (2008, p. 9). Por su parte, Harden (2001, p. 123) describe que:

El mapeo curricular se trata de representar espacialmente los diferentes componentes del currículo para que se pueda ver fácilmente la imagen completa y las relaciones y conexiones entre las partes del mapa. Esta imagen completa es más significativa para el maestro, el alumno o el administrador que la imagen presentada por la colección aleatoria de piezas, que a menudo es lo que tienen.

Los autores citados anteriormente, concuerdan en que -mediante el uso del mapeo curricular, el propósito, el contenido y el diseño del plan de estudios- se evidencian y resultan más transparentes para las personas interesadas: docentes, administradores del currículo, futuros empleadores, estudiantes, padres y madres y entidades externas (como agencias acreditadoras) que requieran consultarlo. Señalan que esta herramienta de representación curricular puede ayudar a guiar el diseño, la mejora del programa, incluida la coherencia, la equidad, la calidad y la eficacia.

Como se observa en la figura 2, Veltri et al. (2011) recurren a una matriz, en la cual los resultados de aprendizaje establecidos del programa, aparecen en la fila superior horizontal; en el caso de las asignaturas o cursos del programa, estos se ubican en la columna izquierda vertical. Puede notarse que en el cruce de las filas y columnas se asigna uno o más resultados de aprendizaje establecidos, representados con una " $\mathrm{X}$ " o 
una "M". Para llevar a cabo este paso, los autores recomiendan acudir a los enunciados de los programas de las asignaturas o cursos, para verificar si cada resultado de aprendizaje establecido se incorporó de forma explícita o implícita en el programa (esto se representa con una $\mathrm{X}$ o una $\mathrm{M}$ en la matriz).

Para efectos de visualizar el alcance y la complejidad del conocimiento y habilidades que se esperan de la asignatura o curso, los autores proponen que cada cruce curso/resultado de aprendizaje previsto se catalogue como introducido (I), enfatizado (E), reforzado (R) o avanzado (A). Por último, en el ámbito de la evaluación, los autores proponen que se efectúe una revisión de las asignaciones circunscritas en los programas, para corroborar si el estudiantado tiene oportunidad de demostrar lo que ha aprendido con respecto a cada resultado de aprendizaje, y, además, verificar si se brinda retroalimentación mediante un mecanismo formal, tal como notas o devoluciones escritas (representado con una " $F$ ").

Figura 2. Matriz de mapeo de los resultados de aprendizaje previstos del programa con respecto a los cursos asociados a éste. Fuente: adaptado de Veltri et al. (2011).

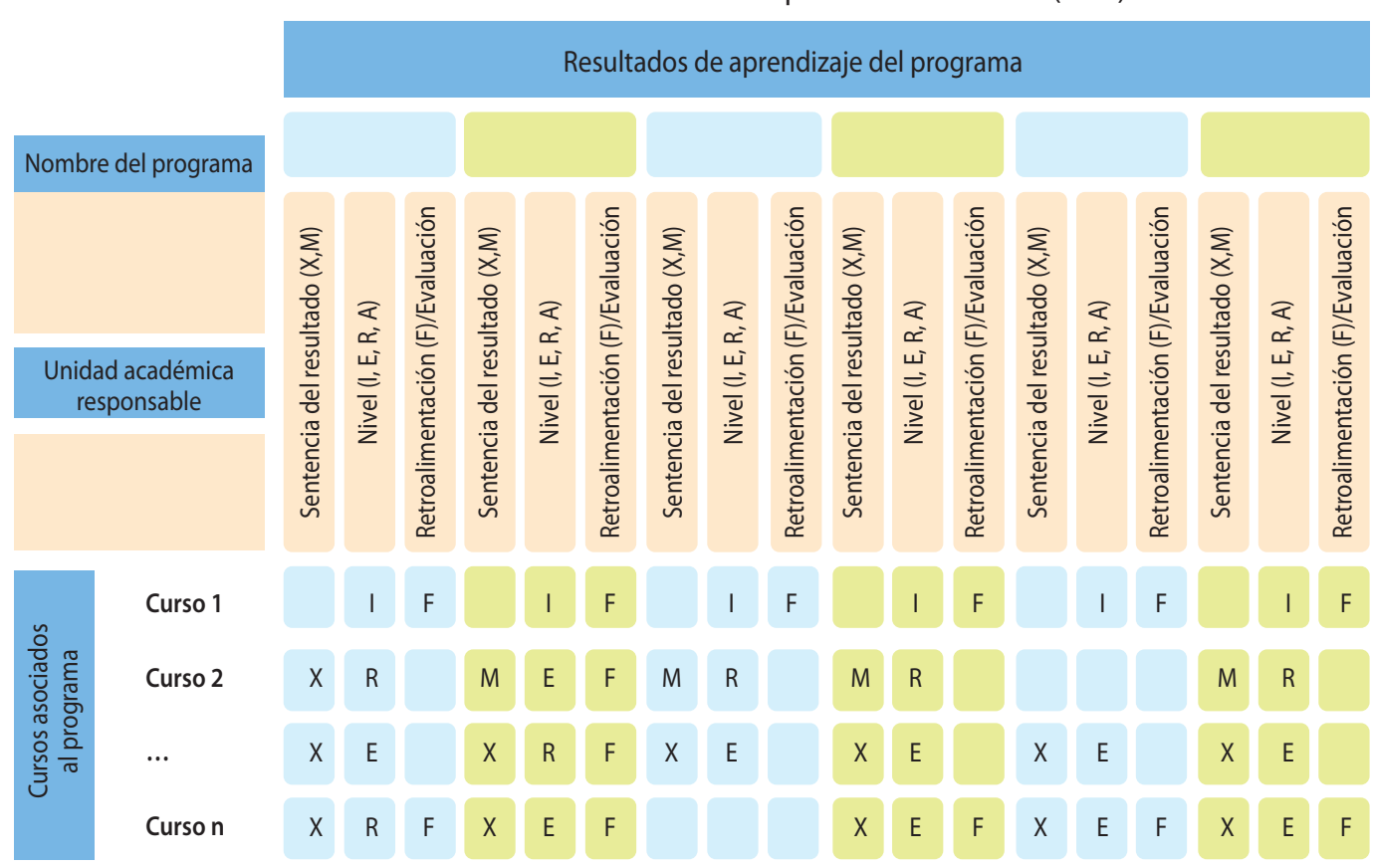

Fuente: Adaptado de Veltri et al. (2011).

Así, el mapeo curricular se puede considerar como un proceso permanente para documentar lo que se enseña en un plan de estudios; de una manera más significativa y está conectado con los resultados de aprendizaje.

Según la literatura revisada, como común denominador se encuentra que para la descripción de los resultados de aprendizaje se parte de una taxonomía, la cual oriente sobre el nivel de conocimiento/ comprensión y el tipo de dominio cognitivo que interesa que el estudiantado logre. En este trabajo se ha encontrado que las taxonomías más utilizadas para esta tarea son las de Bloom, Bloom revisada y SOLO.

Para Bloom, Engelhart, Furst, Hill y Krathwohl (1956) las taxonomías en educación vienen a establecer un orden en las actividades y posibles objetivos que el estudiantado debe alcanzar. Para ello, se establecen niveles de dominio, que conforme el estudiantado los logra más se acerca al resultado del objetivo.

La taxonomía de Bloom fue la primera en ser desarrollada en el campo educativo. En ella, se presentan en forma detallada seis niveles de desarrollo cognitivo (conocimiento, comprensión, aplicación, análisis, 
síntesis y evaluación) y, a su vez, estos cuentan con subcategorías o procesos (Bloom et al., 1956). En esta taxonomía, el nivel de desarrollo cognitivo es móvil, por cuanto puede alcanzarse de forma escalonada. Es decir, para que una tarea sea aprendida, puede requerir de un proceso mental con cierto grado de complejidad, pero posterior a su aprendizaje deja de ser compleja.

Ante la taxonomía de Bloom, hay posiciones encontradas entre los autores. Existen quienes se inclinan a pensar que el conocimiento no se puede encasillar o atomizar en una categoría. Otros como Krathwohl (2002) consideran que es un aporte sustancial al proceso educativo; pues permite establecer un lenguaje común de metas a las personas docentes, para el proceso educativo.

Anderson y Krathwohl (2001) realizaron una revisión de la taxonomía de Bloom y concluyeron que no se trata solo de visualizar el desarrollo cognitivo, sino que esta puede aportar en cuanto a los cuatro tipos de conocimiento que se desea que el estudiantado logre (factual, conceptual, procedimental y metacognitivo). Al respecto, Su, Osisek y Starnes (2005) opinan que la taxonomía de Bloom revisada tiene tres principales beneficios: el primero de ellos, permite que las personas docentes capacitadas en su uso, puedan establecer en su planeamiento la congruencia entre las intencionalidades de la enseñanza, las actividades de aprendizaje y los métodos seleccionados para la evaluación. En segundo lugar, Su et al. (2005) coinciden en que la taxonomía de Bloom revisada puede usarse para planear los procesos de análisis tanto de una clase, como una unidad, módulo o hasta de la asignatura o curso completo. El tercer beneficio, consiste en la oportunidad que pueden tener las personas docentes de incluir objetivos de tipo metacognitivos de manera explícita, con lo cual se puede apoyar al estudiantado en su proceso de construcción de conocimiento.

Por su parte, la taxonomía Observed Learning Outcomes (SOLO por sus siglas en inglés) se diseñó acorde con los diferentes estadios planteados en la teoría del desarrollo cognitivo de Jean Piaget. Es un modelo jerarquizado que representa la forma en que una persona estudiante va adquiriendo diferentes niveles de comprensión, en los cuales cada nivel se convierte en una base sobre la cual se construye aprendizaje adicional (Biggs \& Collis, 1982). Con respecto a la estructura, SOLO contempla cinco niveles: pre-estructural, uni-estructural, multi-estructural, relacional y abstracto extendido (Biggs \& Tang, 2011).

En el nivel 1 que sería el pre-estructural, se parte de la consideración de que el estudiantado no tiene ningún tipo de comprensión; por lo tanto, la información puede utilizarse de forma desorganizada y sin el establecimiento de relaciones para una comprensión integral de un tema o bien, de alguna situación problema. El nivel 2 uni-estructural, se espera que el estudiantado puede establecer conexiones básicas y hasta obvias, en este nivel se busca que la capacidad de recordar y la realización de instrucciones, así como, la identificación de objetos simples, sean tareas que puedan ser fácilmente logradas. En el nivel 3 multi-estructural, el estudiantado debe lograr el abordaje de diferentes temas, pero de forma independiente. Según Biggs y Tang (2011), el estudiantado debe tener la capacidad para poder enumerar, describir, clasificar, combinar, aplicar métodos, estructurar y hasta ejecutar procedimientos. Mientras que en el nivel 4 relacional, ya es capaz de establecer relaciones entre objetos y visualizar de manera integral un fenómeno. En el caso del nivel 5, abstracto extendido, sería el de más alto desarrollo cognitivo. El estudiantado está capacitado para juzgar, teorizar, plantear hipótesis sobre diferentes situaciones al mismo tiempo.

Finalmente, en cuanto a la redacción de resultados de aprendizaje, tanto la taxonomía de Bloom como la de Bloom revisada son las más utilizadas, debido a que proveen verbos, categorizados según el dominio cognitivo, los cuales las personas docentes podrán usar para la redacción de los resultados de aprendizaje (Kennedy, 2006). 


\section{MATERIALES Y MÉTODOS}

El diseño de la investigación fue de corte cualitativo, dado que la intencionalidad fue conocer la perspectiva, opiniones y criterios de las personas participantes sobre diversos aspectos relacionados con el diseño curricular de planes de estudio y asignaturas o cursos bajo el enfoque de resultados de aprendizaje. Según Hernández, Fernández y Baptista (2010), la investigación cualitativa no pretende la generalización de datos sino más bien, el acercamiento a la temática mediante el uso de diversas técnicas de investigación, las cuales permitan interpretar la información. Por otra parte, según el alcance del estudio, se define de tipo exploratorio, dado que es el primer contacto del grupo de investigadores con el tema.

El estudio fue desarrollado en tres etapas: la primera consistió en la indagatoria a nivel bibliográfico bajo el método de revisión de literatura; en una segunda etapa se realizaron tres entrevistas semiestructuradas a un total de cinco expertos entre nacionales e internacionales sobre el tema y finalmente, se efectuó el análisis de la información.

Para la revisión de literatura, se llevó a cabo el proceso metodológico que se describe a continuación:

a) Se definieron criterios para la escogencia de las fuentes, los cuales se sustentaron en los objetivos del estudio. Con respecto al rango de tiempo, por tratarse de un estudio exploratorio, se consideraron publicaciones recientes, pero también las que brindaban un panorama sobre el origen y la evolución de las teorías de interés para la investigación. De la totalidad de fuentes analizadas el $46,15 \%$ fueron publicaciones entre el 2016 y 2020, el 53,85\% restante tiene un rango de publicación entre 1956 y 2015 . En el caso de los tipos de documentos, se incluyeron artículos científicos, libros, guías, tesis, ensayos y ponencias, de los cuales el 63,46\% fue artículos y el 15,38\% libros. En referencia al idioma, se incorporaron textos académicos escritos en inglés y español, con predominio en inglés, con un $88,46 \%$. El cuarto criterio utilizado (el contexto), contempló la selección de los casos de fuentes que tuvieran relación con procesos de diseño curricular universitario y con el enfoque de resultados de aprendizaje.

b) Durante el proceso de búsqueda, se consideraron las siguientes bases de datos, las cuales poseen capacidades web en la localización de documentos, mediante palabras claves. Así, se emplearon: Dialnet, Eric, Google Scholar, Redined, ScienceDirect, Springer Link, Taylor \& Francis Online. También, se efectuaron consultas en los centros de documentación de la Universidad de Costa Rica (UCR) y la Universidad Estatal a Distancia de Costa Rica (UNED).

c) En referencia a los términos de búsqueda, se utilizaron los siguientes: alineamiento constructivo, competencias, currículo basado en resultados de aprendizaje, escritura de resultados de aprendizaje, mapeo curricular, resultados de aprendizaje, taxonomía de Bloom y taxonomía SOLO. Del total de documentos revisados, un 7,69\% se relacionó con alineamiento constructivo, un 9,61\% con competencias, un $11,53 \%$ sobre currículo, un $15,38 \%$ con mapeo curricular, $28,84 \%$ con resultados de aprendizaje, 7,69\% con taxonomía de Bloom y 17,30\% con taxonomía SOLO.

d) Los documentos que cumplían los criterios de inclusión se descargaron y se clasificaron en el gestor Mendeley.

e) Para la sistematización y análisis de las fuentes, se creó una hoja de cálculo en la cual se extrajeron los siguientes datos: referencia en APA, tipo de fuente (primaria, secundaria), tipo publicación (artículo, guía, libro, ponencia, tesis), año de publicación, nombre de la revista, idioma, base de datos en donde se localizó el documento, categoría de análisis (perfil de salida de la persona graduada, evaluación de los aprendizajes, metodología del plan de estudios, malla curricular, resultados de aprendizaje, mapeo curricular, diseño curricular, alineamiento constructivo, revisión curricular, marco de cualificaciones, taxonomías y competencias) y observaciones 
En el momento del análisis, se consideró si el documento tenía relación con alguna de las categorías de análisis definidas, para ello se indicaba si el texto brindaba un aporte principal o aporte secundario. En el caso de la columna de observaciones, se incorporó una síntesis del contenido para efectos de consulta durante el desarrollo de la investigación.

En la segunda etapa de la investigación, se realizaron tres entrevistas a un grupo de cinco personas expertas en el tema, quienes fueron seleccionadas por su experiencia en el diseño curricular con el enfoque de resultados de aprendizaje y, por su trayectoria en el campo de la educación superior. Se aclara que dos de las entrevistas fueron individuales y una grupal, con la participación de tres especialistas. Entre las calidades de las personas entrevistadas están las siguientes:

- Persona investigadora, Universidad Estatal a Distancia, Costa Rica, con experiencia en diseño curricular.

- Docentes investigadores, Universidad de Cádiz y Universidad del País Vasco/Herriko Euskal Universitatea, España, especialistas en evaluación de los aprendizajes.

- Persona investigadora, Universidad de Costa Rica, Costa Rica, con experiencia en capacitación didáctica para docentes universitarios.

Para la entrevista se utilizó un instrumento, el cual consistía en una guía de preguntas que cubría las siguientes categorías: resultados de aprendizaje, alineamiento curricular, mapeo curricular, repercusiones del uso del enfoque de resultados de aprendizaje en los componentes curriculares y uso de taxonomías en la redacción de resultados de aprendizaje. Este instrumento previo a su aplicación fue validado por una persona experta en el área de didáctica y currículo con respecto a la redacción, el contenido y la pertinencia, con una escala de 1 a 5 , donde 1 implicaba deficiente y 5 excelente. La devolución recibida se empleó como parámetro en la mejora del instrumento. Dichas entrevistas se desarrollaron en línea mediante la plataforma Zoom ${ }^{\circledast}$ y, se grabaron (audio e imagen), con el consentimiento de las personas expertas participantes. Estas se transcribieron en una plantilla y, posterior a ello, se elaboró una matriz con las preguntas y los aportes más significativos, con el objetivo de identificar coincidencias y divergencias, lo cual favoreció el análisis y la triangulación de datos con los resultados previamente obtenidos de la revisión de literatura.

\section{DISCUSIÓN DE RESULTADOS}

Seguidamente, se detallan los principales resultados producto de las entrevistas y la revisión de literatura. Para efectos de organización del apartado se presenta la información, según los grandes temas abordados, los cuales son: resultados de aprendizaje, taxonomías, diseño e implementación curricular del enfoque por resultados de aprendizaje, alineamiento constructivo y mapeo curricular.

\section{Resultados de aprendizaje}

En cuanto al tema de resultados de aprendizaje, a partir de las entrevistas se encontró que, todas las personas expertas conocen el enfoque de resultados de aprendizaje. Cuatro de ellas coinciden en que este enfoque busca un cambio en los modelos pedagógicos. En estos casos, el estudiantado se convierte en el centro del proceso educativo. Al respecto una de las personas entrevistadas indicó: "[...] un enfoque de resultados de aprendizaje es un enfoque que se centra en el proceso de aprendizaje y que privilegia el resultado que demuestra el estudiante al finalizar un proceso de formación". (Persona entrevistada uno). 
Una de las personas nacionales entrevistadas indica que el enfoque de resultados de aprendizaje no es novedoso; sin embargo, desde el Proceso de Bolonia, se dio el resurgimiento de este modelo, lo cual es muy positivo, puesto que a la fecha, la didáctica se ha mantenido en la línea tradicional, la persona docente es el centro del proceso o bien los contenidos. Al respecto, una de las personas entrevistadas destacó el tema, al mencionar que:

[...] del enfoque de resultados de aprendizaje, lo de Bolonia y el proyecto de Tuning [...] se dice que se debe optar por un enfoque de aprendizaje en los planes de estudios, que explicite los logros o las metas que debe tener el estudiante al final de cierto proceso formativo. (Persona entrevistada cinco)

En relación con la participación de las personas expertas en el proceso de elaboración de marcos de cualificación orientado desde el enfoque de resultados de aprendizaje, se encontró que solamente una de ellas ha tenido este tipo de experiencia; pues participó en la elaboración del MCESCA.

Por su parte, los expertos europeos han participado en el diseño de resultados de aprendizaje para carreras y por asignaturas. Según indicaron, en la revisión de los programas de las asignaturas, las personas docentes siguen diseñando los resultados como objetivos tradicionales; por lo tanto, la formación y la actualización docente es una tarea que debe continuar, pues resulta novedoso en este momento. Para Jenert (2014) el desarrollo de los planes de estudio orientados a resultados requiere que la persona docente sincronice y ordene los cursos entre sí, de manera que apoye el logro de los resultados. También, plantea que debe existir una reconciliación entre la cultura hacia lo disciplinar que identifica al profesorado y la implementación de los programas de estudio orientados a resultados.

Respecto al tema anterior expuesto, se encuentra en la revisión de literatura que, en la última década, se han desarrollado un número considerable de publicaciones académicas sobre política e informes, los cuales responden a cuestiones asociadas con la definición de resultados de aprendizaje, módulos, sistemas de créditos, en general, sobre el cuerpo de conceptos. Keller (2006); Marian (2007); Schaper, Schlomer y Paechter (2013); Webler (2005); citados por Jenert (2014).

Jenert (2014), indica que las reformas curriculares se mantienen en el nivel de cambios estructurales, en lugar de influir de manera sostenible en las prácticas de enseñanza y aprendizaje de forma que apoye el logro de los objetivos educativos subyacentes.

Por otro lado, Jenert (2014) cita el trabajo de Barr y Tagg (1995) titulado "El paso de la enseñanza al aprendizaje", en ese caso, se ejemplifica una currícula en la educación superior diseñada mediante una compilación de conocimientos que responden, puntualmente, a las disciplinas involucradas versus aquellos programas de la educación superior "que pasaron" a definir resultados de aprendizaje previstos en función del estudiantado. Esta segunda alternativa conlleva a que la enseñanza debe orientarse más hacia las necesidades del estudiantado y, debe emplearse la información sobre los procesos de aprendizaje para apoyarlo. Así, Jenert (2014) sustentado en el trabajo de Biggs (2003), propone que el diseño curricular debe apuntar al alineamiento entre los resultados de aprendizaje con las metas, estructuras de estudio, procesos de enseñanza-aprendizaje y procedimientos de evaluación. El autor citado indica que la escasez de publicaciones respecto a la implementación, gestión y desarrollo de programas de estudio es un reto por considerar.

En cuanto a la redacción de los resultados de aprendizaje, Kennedy (2006) propone que la redacción debe ser clara y concisa; además, debe permitir su evaluación. Para ello, recomienda el uso de la taxonomía de Bloom o la de Bloom revisada, dado que provee en cada nivel, verbos suficientes para orientar a la persona docente en el proceso de redacción de los resultados de aprendizaje. Sin embargo, pueden utilizarse otras taxonomías, siempre y cuando orienten a la persona docente en la selección del verbo de acción. 
Todo resultado de aprendizaje tiene una estructura por seguir: verbo de acción más complemento más frase que provea información del contexto. Aunado a lo anterior, Kennedy (2006) refiere que existen dos tipos de resultados de aprendizaje. El primero se refiere a los que son visibles en los componentes curriculares de la unidad, el módulo o el programa de la asignatura o curso y se reflejan tanto en los contenidos, las actividades de aprendizaje y la evaluación. El segundo está compuesto por los que están contenidos y son visibles en el plan de estudios y no se detallan en la unidad, el módulo o el programa de la asignatura o curso. Sin embargo, se refieren a la calidad del desempeño del futuro profesional. Por lo tanto, este tipo de resultados está ligado a aquellos de tipo afectivo y actitudinal.

Por su parte, Biggs y Tang (2011) proponen que la redacción de los resultados de aprendizaje de un curso debe considerar la inclusión de un verbo que refleje el nivel de compresión, el contenido del tema que el verbo abordará y el contexto de la disciplina en la cual se implementará el verbo. Un ejemplo de resultado de aprendizaje que utiliza la taxonomía de Bloom en el nivel de conocimiento, según la propuesta de Kennedy (2006, p. 26) es el siguiente:

Identificar las implicaciones éticas en investigaciones científicas

Además, Kennedy (2006), Biggs y Tang (2011) indican que, en el caso de asignaturas o cursos, se deben proponer como máximo de cinco a seis resultados para su cumplimiento en el periodo académico, independientemente de la cantidad de tópicos o contenidos contemplados.

\section{Taxonomías}

En la revisión de literatura se halla que las taxonomías más utilizadas para la redacción de resultados de aprendizaje son la de Bloom, la de Bloom revisada y la de SOLO de Biggs. La selección de las taxonomías obedece a la jerarquía del desarrollo cognitivo en cada uno de los niveles, así como en los verbos de acción que se encuentran en cada uno, lo cual proporciona a la persona docente elementos para la construcción de los resultados de aprendizaje, de manera que les oriente en esta tarea. Así, una de las personas entrevistadas opinó respecto a la taxonomía de Bloom revisada:

[...] con que un profesor o profesora conozca esa taxonomía, es muy valiosa para poder redactar buenos resultados de aprendizaje $y$, además, teniendo en cuenta que se intenta ir a los de más alto nivel, los que exigen un proceso mental superior, no solamente el recordar. (Persona entrevista tres)

Respecto al uso de las taxonomías las personas consultadas refieren que es necesario el uso de estas, para redactar los resultados de aprendizaje. Sin embargo, no está restringido a una en particular, la elección de esta queda a criterio de las personas docentes, quienes deben tener en consideración que, dicha taxonomía esté alineada con los principios epistemológicos destacados en el plan de estudios de la carrera. También coinciden en que la Taxonomía de Bloom facilita gran cantidad de verbos por nivel, lo cual puede orientar la tarea de la redacción de los resultados de aprendizaje (Kennedy, 2006).

Sin embargo, una de las personas entrevistadas indica que el uso de la Taxonomía de Bloom limita y tiende a encasillar, prefiere que se utilice Bloom revisado o SOLO, esta última le parece importante pues no se centra en contenidos sino en niveles de desarrollo cognitivo. Al respecto, autores como Brabrand y Dahl (2009a), coinciden con esta apreciación. Una de las personas entrevistadas mencionó lo siguiente: 
[...] las otras son más complicadas, no quiere decir que sean peores, pero intentar crear resultados de aprendizaje basándonos en la taxonomía SOLO, por ejemplo, a nosotros que teóricamente somos expertos en educación y nos dedicamos a eso, nos ha generado muchísimas discusiones. Sin embargo, cuando nos centramos en la taxonomía de Bloom renovada eso lo entendemos bastante bien y tenemos mucho menos discusiones. (Persona entrevistada cuatro)

\section{Diseño e implementación curricular del enfoque por resultados de aprendizaje}

Las personas entrevistadas afirman que es fundamental que la persona docente participante en el diseño curricular y la implementación del plan de estudios, desde el enfoque por resultados de aprendizaje, tenga en cuenta que se trata de un cambio de visión, en el cual el contenido no es lo principal, ni la memoria o la repetición de conceptos. Se trata del desarrollo de habilidades en el estudiantado y quien debe orientar hacia la evaluación formativa es la persona docente (Kennedy, 2006). Desde el inicio del proceso educativo, consideran que los resultados de aprendizaje deben ser de conocimiento tanto del estudiantado como de la persona docente, igual que los criterios e instrumentos de evaluación. Así, resaltan la importancia de la evaluación de los aprendizajes; pues debe darse un cambio y, se espera que se dejen de lado las pruebas objetivas y más memorísticas. Una de las personas entrevistadas, además comentó:

[...] nosotros nos movemos bastante en el contexto de la evaluación de la educación superior, entonces, a nosotros nos parece que la base fundamental donde se tiene que planificar todos los programas y todas las asignaturas es sobre el concepto de las tareas de evaluación, a través de las tareas de evaluación se deberían conseguir esos resultados de aprendizaje. (Persona entrevistada dos)

\section{Alineamiento constructivo}

Al efectuar la consulta sobre el concepto e implementación de alineamiento curricular, una de las personas entrevistadas indica haber trabajado con el alineamiento constructivo de John Biggs; asimismo, el grupo de investigadores europeo concuerdan en que conocen del concepto, el cual comprenden como la convergencia coherente entre la evaluación, los resultados de aprendizaje y las tareas de aprendizaje/enseñanza.

Por otra parte, solamente dos de las personas han escuchado del concepto de alineamiento curricular. Una de ellas indica que vincula el término con el diseño curricular específicamente con la coherencia y la relación entre los elementos curriculares, tanto en el planteamiento de un plan de estudios como en el de las asignaturas o cursos. Mientras que la otra mencionó que el alineamiento lo ha trabajado más desde la ejecución del plan de estudios y no desde el diseño curricular.

\section{Mapeo curricular}

En la revisión de literatura se halla que diversos autores definen el mapeo curricular como una herramienta con la cual puede evidenciarse si los diferentes componentes curriculares se alinean. Indican, además, que la imagen que proporciona el mapeo curricular es importante para las diferentes personas que interactúan con el currículo (estudiantes, docentes, administradores, empleadores). Rawle, Bowen, Murck y Hong (2017) consideran también que la herramienta se puede emplear para identificar los resultados de aprendizaje a lo largo del currículo. 
De esta forma, al consultarle a los entrevistados respecto a este concepto de mapeo curricular, el grupo de investigadores europeo y una de las expertas nacionales no conocen del concepto, pero lo han utilizado de manera parcial con matrices $u$ otros instrumentos para saber la relación que hay entre los elementos curriculares, por ejemplo: los rasgos del perfil con las asignaturas o cursos y con los objetivos. Según indicó una de las especialistas, lo que se busca es recabar información de en qué medida se están consiguiendo esos resultados de aprendizaje. En ese sentido, una de las personas entrevistadas indicó:

Elaborar un portafolio reflexivo, esto es el mapeo a nivel de resultados de aprendizaje, competencias y productos. Faltaría incluir qué instrumentos de evaluación utilizamos para valorar cada uno de esos productos, como rúbricas o escalas de valoración. Además, en esas rúbricas y en esas escalas especificamos cuales son los resultados de aprendizaje que se están valorando y con qué competencias están relacionadas, de tal manera que podemos, a través de los propios instrumentos de evaluación recabar información de en qué medida se están consiguiendo esos resultados de aprendizaje. Esto sería un mapeo parcial porque evidentemente no contempla todos los elementos. (Persona entrevistada tres)

Una de las personas expertas nacionales manifiesta que conoce del mapeo curricular y ejemplifica el uso de las matrices para mapear esa coherencia entre los elementos curriculares, e indica que esto va muy de la mano con el concepto de alineamiento. Esta persona manifestó lo siguiente:

[...] el mapeo curricular de hecho se utiliza mucho para las evaluaciones y el seguimiento, de manera que al aplicarlo se utilizan las matrices para mapear esa coherencia y eso va muy de la mano incluso con lo que acabamos de hablar del alineamiento. (Persona entrevistada cinco)

\section{SÍNTESIS Y REFLEXIONES FINALES}

Tanto a nivel regional como nacional, se vienen desarrollando esfuerzos orientados a incorporar el enfoque de resultados de aprendizaje en la currícula de la educación superior, prueba de ello es la elaboración del Marco de Cualificaciones para la Educación Superior Centroamericana (MCESCA), con el cual se espera priorizar en su implementación. Estos marcos de cualificaciones orientan un camino o ruta para buscar estándares o indicadores regionales, los cuales promuevan la movilidad, el reconocimiento y la apertura de espacios a nuevas opciones de aprendizaje.

En relación con el primer objetivo se hallaron elementos importantes por considerar en el diseño curricular de planes de estudios y asignaturas o cursos desde el enfoque de resultados de aprendizaje, entre los que se destacan:

La importancia de que exista coherencia entre resultados de aprendizaje que se definan para un plan de estudios y los que se plantean para las asignaturas o cursos. Es necesario que, en la descripción de asignaturas o cursos, se evidencie la articulación y la congruencia con los que fueron planteados a nivel del plan de estudios en los diferentes componentes curriculares, de lo contrario puede repercutir en el planteamiento de dos propuestas desarticuladas. Esta situación podría ser perjudicial para el proceso formativo del estudiantado; pues existe el riesgo de que se dificulte la incorporación de los resultados de aprendizaje en la oferta educativa planteada.

El logro de la coherencia mencionada en el párrafo anterior, requiere contar con un diseño curricular donde sus diferentes componentes hayan sido establecidos para el alcance de los resultados de aprendizaje; según los insumos de la revisión de literatura, es lo que se conoce como alineamiento constructivo. 
De acuerdo con la teoría del alineamiento constructivo, los resultados de aprendizaje previstos en una asignatura o curso deben converger con las actividades de enseñanza/aprendizaje y en las tareas de evaluación Esta teoría considera como herramienta práctica para sus fines, el mapeo curricular. De esta manera, el mapeo curricular viene a ser el proceso permanente, el cual evidencia que lo que se enseña en el plan de estudios, está relacionado en forma directa con los resultados de aprendizaje.

Para el diseño o la actualización de un plan de estudios por resultados de aprendizaje, se requiere de la participación de la persona docente que asumirá su implementación; quien, a su vez, debe adquirir las capacidades y los conocimientos en relación con el enfoque, tarea prioritaria que debe atenderse previo a la planificación o ejecución de la oferta educativa; pues a nivel metodológico y evaluativo este enfoque plantea un cambio en el rol de la persona docente.

En cuanto al enfoque de resultados de aprendizaje, este se centra en el estudiantado, figura protagónica de su proceso formativo. Por ello, es que la persona docente debe darle a conocer los logros, las actuaciones, las actividades y la evaluación que se espera lleven a cabo en la asignatura o curso, así como informarle de sus avances en el proceso de aprendizaje.

La incorporación de este enfoque en el diseño curricular de los planes de estudios, conlleva el apoyo imprescindible de las instancias institucionales superiores y, el involucramiento del estudiantado, para alcanzar con éxito las prácticas de enseñanza/aprendizaje propuestas. El desarrollo de políticas institucionales que apoyen la movilidad y, a la atención a la diversidad estudiantil. Así como la promoción de la calidad en los procesos de enseñanza/aprendizaje, por cuanto, al incorporar este enfoque, se esperaría elevar los estándares de calidad en la educación superior.

El criterio de las personas expertas resulta coincidente con los aspectos destacados en los párrafos anteriores de este apartado. Sin embargo, fueron enfáticas en mencionar que la incorporación del enfoque de resultados de aprendizaje, permite un cambio metodológico en el rol tradicional de la persona docente; pues se espera que aplique novedosas opciones didácticas para que el estudiantado alcance el cumplimiento de los resultados de aprendizaje. De esta forma, una de las tareas que se requiere, es que el trabajo docente se realice de manera colaborativa; es decir, romper la disciplinariedad para dar paso a procesos de enseñanza interdisciplinarios.

Otro aspecto que se destacó en las entrevistas es el cambio de mentalidad que se debe dar en los procesos de diseño curricular. Según las personas entrevistadas, resulta necesario visualizar de forma conjunta el proceso de elaboración del plan de estudios, con el diseño de asignaturas o cursos, para conseguir el alineamiento constructivo entre los componentes.

Finalmente, la revisión de literatura permite concluir que la incorporación del enfoque de resultados de aprendizaje en el diseño curricular de planes de estudio y diseños de asignaturas o cursos, es una tendencia que cobra fuerza en el contexto actual. Sin embargo, las investigaciones sobre su impacto son todavía incipientes; por lo tanto, la efectividad en los procesos de aprendizaje del estudiantado es un tema que deberá investigarse.

Por otra parte, la labor realizada por las personas que se involucran en el diseño de un plan de estudios, se ha desarrollado tradicionalmente con recursos manuales o automatizados como hojas de cálculo. No obstante, el uso de una herramienta informática no ha sido la constante o, al menos, no se encontró información de ello en la revisión de literatura. Dado lo anterior, se evidencia la factibilidad y viabilidad de desarrollar una herramienta informática, para realizar el mapeo del currículo, como mecanismo de seguimiento y visualización, el cual posibilite determinar dónde, cuándo y cómo los resultados de aprendizaje son desarrollados y evaluados dentro del plan de estudios. Además, que provea un panorama integral de los componentes del currículo a todos los actores involucrados como: comisión de diseño curricular, asesores curriculares, personas docentes, estudiantado, pares evaluadores externos y empleadores. 
Agencia Nacional de Evaluación de la Calidad y Acreditación. (s.f.). Guía de apoyo para la redacción, puesta en práctica y evaluación de los resultados de aprendizaje. http://www.aneca.es/content/ download/12765/158329/file/learningoutcomesv02.pdf

Alfauzan, A. H, \& Tarchouna, N. (2017). The Role of an Aligned Curriculum Design in the Achievement of Learning Outcomes. Journal of Education and E-Learning Research, 4(3):81-91. doi: 10.20448/ journal.509.2017.43.81.91

Anderson, L. W., \& Krathwohl, D. R. (2001). A taxonomy for learning teaching and assessing: a revision of Bloom's taxonomy of educational objectives. Pearson Education.

Australian qualifications framework. (2013). Australian Qualifications Framework Council Australian Qualifications Framework Council. Recuperado de https://www.aqf.edu.au/sites/aqf/files/aqf2nd-edition-january-2013.pdf

Bloom, B. S., Engelhart, M. D., Furst, E. J., Hill, W. H., \& Krathwohl, D. R. (1956). Taxonomy of Educational Objectives: Handbook I Cognitive Domain. Estados Unidos: Longmans, Green and Co. Ltd.

Biggs, J. B., \& Collis, K. F. (1982). Evaluating the quality of learning: The SOLO taxonomy (Structure of the Observed Learning Outcome). Estados Unidos: Academic Press.

Biggs, J., \& Tang, C. (2011). Teaching for quality learning at university (4th ed.). Inglaterra: McGraw-Hill.

Bohlinger, S. (2008). Las competencias: elemento básico del Marco Europeo de cualificaciones. Revista Europea de Formación Profesional, 42/43:103-120. Recuperado de https://dialnet.unirioja.es/descarga/articulo/2556384.pdf

Bolaños, G. \& Molina, Z. (1997). Introducción al currículo. San José, Costa Rica: EUNED.

Bologna Working Group on Qualifications Frameworks. (2005). A framework for qualifications of the European higher education area. Ministry of Science, Technology and Innovation. Recuperado de http://ecahe.eu/w/images/7/76/A_Framework_for_Qualifications_for_the_European_Higher_ Education_Area.pdf

Brabrand, C., \& Dahl, B. (2009a). Using taxonomy to analyze competence progression of university science curricula. Higher Education, (59):531-549. doi: 10.1007/s10734-009-9210-4

Brabrand, C., \& Dahl, B. (Noviembre,2007 b). Constructive alignment and the SOLO taxonomy: a comparative study of university competences in computer science vs. Mathematics. En Seventh Baltic Sea Conference on Computing Education Research Koli Calling, Parque Nacional Koli, Finlandia. Recuperado de http://itu.dk/people/brabrand/koli-2007-keynote.pdf

CEDEFOP. (2017). Defining, writing and applying learning outcomes: a European handbook. Publications Office of the European Union. doi: 10.2801/566770

Clemente Linuesa, M. (2010). Diseñar el currículum. Prever y representar la acción. En: Gimeno Sacristán, J. (Ed. de la serie), Saberes e incertidumbres sobre el currículum. https://www.torrossa. com/en/resources/an/2952233

Cohen, S. A. (1987). Instructional Alignment: Searching for a Magic Bullet. Educational Researcher, 16(8):16-20. doi:10.3102/0013189X016008016

Consejo Superior Universitario Centroamericano. (2018). Marco de cualificaciones para la educación superior centroamericana (MCESCA): resultados de aprendizaje esperados para los niveles técnico superior universitario, bachillerato universitario, licenciatura, maestría y doctorado. Editorial Serviprensa. Recuperado de http://www.csuca.org/docs-csuca/libros/Marco\%20de\%20cualificaciones\%20 para\%20la\%20educacion.pdf

Coll, C. (1997). Psicología y currículum: Una aproximación psicopedagógica a la elaboración del currículum. México: Paidós Mexicana. 
Comisión Europea. (2009). El Marco Europeo de Cualificaciones para el aprendizaje permanente ( EQF-MEC). Educación y Cultura, 1-20. doi:10.2766/14724

Harden, R. M. (2001). AMEE Guide No. 21: Curriculum mapping: a tool for transparent and authentic teaching and learning. Medical Teacher, 23(2):123-137. Recuperado de https://doi.org/10.1080/01421590120036547

Hernández, R., Fernández, C. \& Baptista, P. (2010). Metodología de la investigación. México: McGraw-Hill Interamericana.

Jenert, T. (2014). Implementing Outcome-Oriented Study Programmes at University: The Challenge of Academic Culture. Zeitschrift Für Hochschulentwicklung, 9(2). doi: 10.3217/zfhe-9-02/02

Krathwohl, D. R. (2002). A Revision of Bloom's Taxonomy: An Overview. Theory Into Practice, 41(4):212218. http://www.jstor.org/stable/1477405

Kennedy, D. (2006). Redactar y utilizar resultados de aprendizaje. Un manual práctico. https://cora.ucc.ie/ handle/10468/1613

Kennedy, D., \& McCarthy, M. (2016). Learning Outcomes in the ECTS Users' Guide 2015 Some Areas of Concern. Journal of the European Higher Education Area, 3.

Kopera-Frye, K., Mahaffy, J., \& Svare, G. M. (2008). The map to curriculum alignment and improvement. Collected Essays on Learning and Teaching, 1. doi: 10.22329/celt.v1i0.3171

Posner, J. (2007). Análisis del currículo. México: McGraw-Hill Interamericana.

Rawle, F., Bowen, T., Murck, B., \& Hong, R. (2017). Curriculum mapping across the disciplines: differences, approaches, and strategies. Collected Essays on Learning and Teaching, 10. doi: 10.22329/celt.v10i0.4765

Su, M., Osisek, P. J., \& Starnes, B. (2005). Using the Revised Bloom's Taxonomy in the Clinical Laboratory. Nurse Educator, 30(3):117-122. doi:10.1097/00006223-200505000-00014

Thompson, T. S. (2011). Bologna to Berlin 1999-2003: The initial steps of the Bologna Process and creation of the European Higher Education Area. (Publicación No. 3485868). [Disertación doctoral, University of Pittsburgh]. ProQuests Dissertations \& Theses.

Veltri, N. F., Webb, H. W., Matveev, Alexei G, \& Zapatero, E. G. (2011). Curriculum Mapping as a Tool for Continuous Improvement of IS Curriculum. Journal of Information Systems Education, 22(1):31-42. http://jise.org/volume22/n1/JISEv22n1p31.html

Wijngaards-de Meij, L. \& Merx, S. (2018). Improving curriculum alignment and achieving learning goals by making the curriculum visible. International Journal for Academic Development, 23(3):219-231. doi.org/10.1080/1360144X.2018.1462187 